\title{
ON EFFECTIVE MODULI OF AN ELASTIC BODY CONTAINING PERIODICALLY DISTRIBUTED VOIDS*
}

BY

\author{
S. NEMAT-NASSER AND M. TAYA** \\ Northwestern University
}

\begin{abstract}
For an elastic body containing periodically distributed voids, several effective techniques are presented which can be used to obtain the effective elastic moduli with any desired degree of accuracy. The results include the effects of void geometry as well as void interactions. For a body containing spherical voids, numerical results are presented and compared with those obtained by other methods.
\end{abstract}

1. Introduction. Estimates of the overall elastic moduli of an elastic solid which contains voids or cracks are of considerable theoretical and practical interest. In view of this, different methods have been developed by various authors. Most approaches fall into the following classes: (1) self-consistent model, (2) variational approach, (3) statistical method, and (4) exact solutions.

What is now known as the self-consistent method has been used by MacKenzie [1] and later Kerner [2] to estimate the elastic and thermoelastic properties of a solid containing spherical voids [1] and a composite solid [2]. In this approach, say, the void is surrounded by a matrix shell which in turn is surrounded by an unbounded porous material. The elastic moduli derived by these authors $[1,2]$ turn out to be the same as the lowest upper bound later obtained by Hashin and Shtrikman [3] by a variational approach. Hill [4] and Budiansky [5] have used the self-consistent method to estimate the elastic moduli of composites; where the inclusion is regarded as being embedded in an unbounded medium which has the same elastic properties as those of the composite and which is subjected to the prescribed uniform loading at infinity. Then Eshelby' [6] solution for an ellipsoidal inclusion embedded in an unbounded elastic medium 's used to estimate the final results. Hill $[4$, p. 220] points out that the self-consistent method is "unreliable under extreme conditions", i.e. for voids and rigid inclusions, "exc. " when the dispersed phase is sufficiently dilute". It should be mentioned that the seli .....sistent method had been used earlier by Hershey [7] and Kröner [8] to construct a model for polycrystalline materials.

* Received November 13, 1979; revised version received February 27, 1980. The authors wish to thank Dr. M. Hejazi who helped in obtaining the numerical results. This work has been supported by the National Science Foundation under Grant No. ENG76-03921 to Northwestern University.

** Present address: Department of Mechanical and Aerospace Engineering, University of Delaware, Newark, Delaware, 19711. 
Within the framework of extremum principles in elasticity and by introducing a comparison model and a polarization stress, Hashin and Shtrikman [3] derive bounds on the effective elastic moduli of composites. In the case of voids, their method yields only the lowest upper bound, the corresponding highest lower bound becoming zero.

In actual cases void distribution and void geometry are statistical in character, and therefore must be regarded as randomly varying quantities. To account for this, Willis [9] has generalized Hashin and Shtrikman's [3] method, and has discussed a two-point correlation function for composites of this kind. In an earlier work [10] Christoffersen dealt with randomly distributed voids or inclusions in an infinite elastic medium, assuming a constant transformation strain within each void or inclusion. Recently, Afzali and Nemat-Nasser [11] have estimated the elastic moduli for a solid containing periodically distributed voids by calculating the "effective" body forces which must be placed at the center of each void in order to render the void surface traction-free. In this calculation the interaction effects are taken into account to a large extent. However, the results are not exact, although they show considerable improvement on various previous results. This will be discussed later on.

When the voids or inclusions have simple geometries and are periodically distributed, one may attempt to construct exact solutions. Eshelby's [6] results for an ellipsoidal inclusion in an infinite medium are of this kind, where the periodicity length can be regarded as infinity. Eshelby introduces the concept of transformation strain which, in the case of one ellipsoidal inclusion in an infinite medium, turns out to be constant. For a body containing periodically distributed voids and inclusions, one may effectively employ the concept of transformation strain which, however, will no longer be a constant tensor. On the other hand, because of periodicity, one may confine attention to a unit cell, expand the transformation strain as well as other field quantities in appropriate Fourier series, and then construct an integral equation for the corresponding unknown transformation strain. Then this integral equation may be solved by various schemes. For an elastic solid containing periodically distributed voids of the same geometry, we have carried out in this paper the program outlined above.

In Sec. 2 we exploit the concept of transformation strain and obtain an integral equation for this quantity, where the corresponding integral extends over the void volume only. The integral equation involves an infinite series. In Sec. 3 we examine the convergence of this series and present several approximate and one exact method of solution. In addition, we give several illustrative examples. All calculations are performed for spherical voids arranged in a simple cubic lattice; however, generalization for other void geometries is mentioned. In Sec. 4 we outline the variational approach for estimating the overall elastic moduli, use the results of Sec. 3, and obtain overall moduli for some special cases. We then compare our results with those of other authors when the Poisson ratio of the matrix is taken equal to 0.3 .

2. Formulation. Consider an infinitely extended isotropic elastic body which contains periodically distributed voids of common geometry and dimensions, in such a manner that the body may be regarded as a collection of unit cells of identical dimensions, each containing one or several voids. In this work the unit cell is assumed to be a parallelepiped with dimensions $\Lambda_{i}$ measured along the rectangular Cartesian coordinate axes $x_{i}, i=1,2,3$. The body is subjected to the overall average far-field stresses $\sigma_{i j}^{0}$, as 


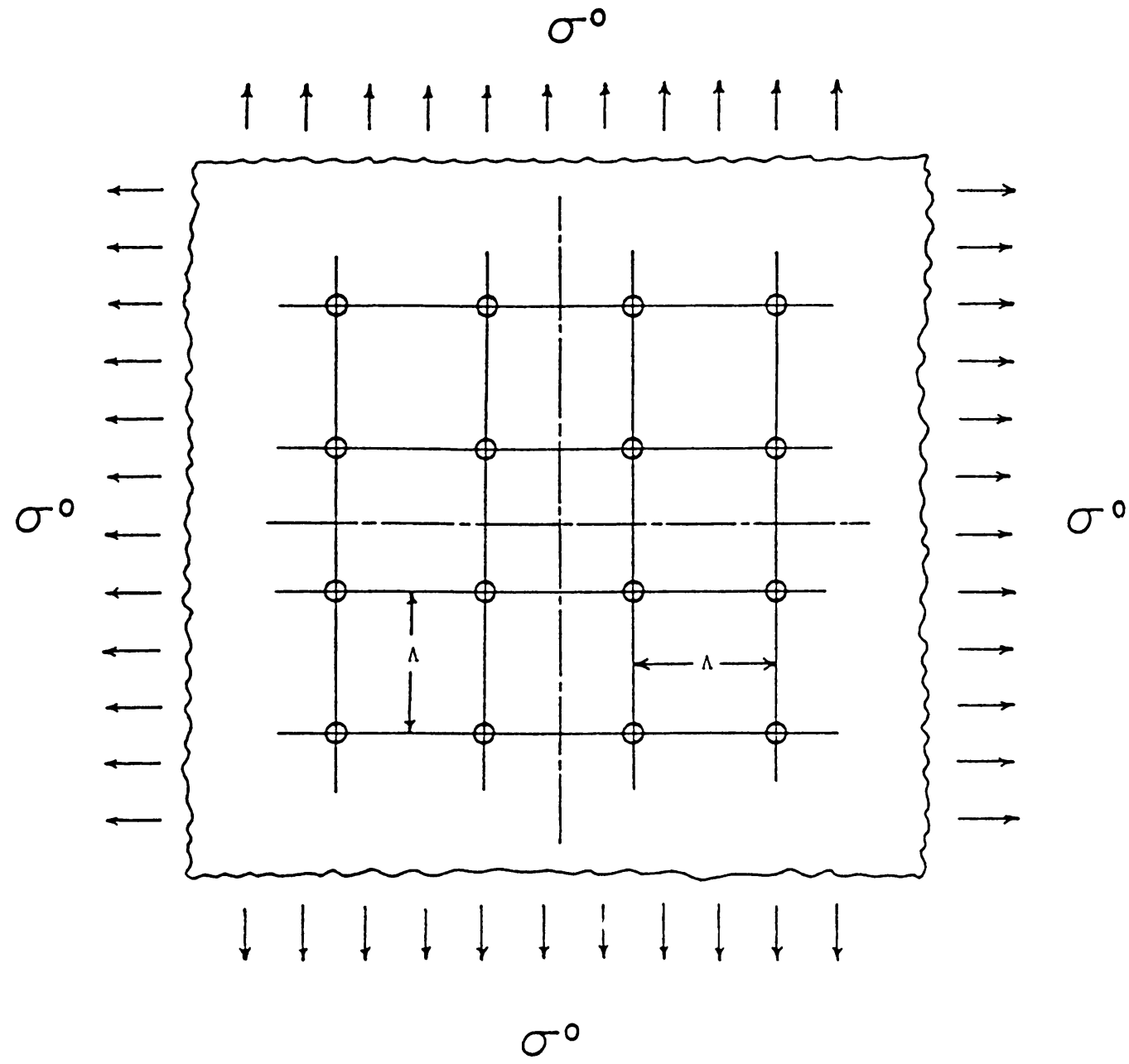

FIG. 1.

schematically shown in Fig. 1 for the special case of spherical voids; here and in the sequel all subscripts take on the values 1,2,3, and repeated indices are summed over the same values unless stated otherwise. In the absence of any voids (homogeneous solid) the corresponding strain field would be given by $C_{i j k l} \varepsilon_{k l}^{0}=\sigma_{i j}^{0}$, where

$$
C_{i j k l}=\lambda \delta_{i j} \delta_{k l}+\mu\left(\delta_{i k} \delta_{j l}+\delta_{i l} \delta_{j k}\right),
$$

and where $\lambda$ and $\mu$ are the Lamé constants for the matrix, $C_{i j k l}$ being the corresponding elasticity tensor; in (2.1) $\delta_{i j}$ is the Kronecker delta. The existence of voids disturbs the homogeneous stress and strain fields, and the total stress field can be expressed as

$$
\sigma_{i j}^{T}=\sigma_{i j}^{0}+\sigma_{i j}=C_{i j k l}\left(\varepsilon_{k l}^{0}+\varepsilon_{k l}\right) \text { in } D-\Omega,
$$

where $\sigma_{i j}=C_{i j k l} \varepsilon_{k l}$ is the perturbation stress field introduced by the voids, the corresponding displacement perturbation being $u_{i}$, and hence $\varepsilon_{i j}=\frac{1}{2}\left(u_{i, j}+u_{j, i}\right)$; a comma 
followed by an index denotes partial differentiation with respect to the corresponding coordinate. In (2.2) $D$ denotes the domain occupied by a typical unit cell (parallelepiped) of dimensions $\Lambda_{i}$ and $\Omega$ is the domain occupied by the cavities contained in that unit cell.

The geometric periodicity implies the periodicity of the perturbation field $\sigma_{i j}, \varepsilon_{i j}$, and $u_{i}$. Moreover, within the region $\Omega$ the total stress field must vanish. To assure this, one may introduce a transformation strain $\varepsilon_{i j}^{*}$ which is identically zero in $D-\Omega$, and is a periodic function ${ }^{1}$ of $\mathbf{x}$. In each cavity within a cell, the total stress must vanish, i.e.

and hence

$$
\sigma_{i j}^{T}=C_{i j k l}\left(\varepsilon_{k l}^{0}+\varepsilon_{k l}-\varepsilon_{k l}^{*}\right) \equiv 0 \text { in } \Omega
$$

$$
-\sigma_{i j}^{0}=C_{i j k l}\left(\varepsilon_{k l}-\varepsilon_{k l}^{*}\right) \text { in } \Omega
$$

The total stress must satisfy the equilibrium equation $\sigma_{i j, j}^{T}=0$, and since $\varepsilon_{i j}^{0}$ is a constant field, (2.3) yields

$$
\left[C_{i j k l}\left(\varepsilon_{k l}-\varepsilon_{k l}^{*}\right)\right]_{j}=0 \text { in } \Omega \text {. }
$$

Because of the periodicity, the fields $u_{i}$ and $\varepsilon_{i j}^{*}$ can be expressed in the Fourier series as ${ }^{2}$

$$
\begin{aligned}
u_{k}(\mathbf{x}) & =\sum_{n_{j}=0}^{ \pm \infty} \hat{u}_{k}(\xi) e^{i \xi \cdot \mathbf{x}}, \quad \varepsilon_{k l}^{*}(\mathbf{x})=\sum_{n_{j}=0}^{ \pm \infty} \hat{\varepsilon}_{k l}^{*}(\xi) e^{i \xi \cdot \mathbf{x}}, \\
\hat{\varepsilon}_{k l}(\xi) & =\frac{1}{\Lambda_{1} \Lambda_{2} \Lambda_{3}} \int_{D} \varepsilon_{k l}\left(\mathbf{x}^{\prime}\right) e^{-i \xi \cdot \mathbf{x}^{\prime}} d \mathbf{x}^{\prime}, \quad \xi_{j}=2 \pi n_{j} / \Lambda_{j} \text { (no sum on } j \text { ), } \\
n_{j} & =0, \pm 1, \pm 2, \ldots, \quad i=\sqrt{ }-1, \quad k, l, j=1,2,3,
\end{aligned}
$$

From $(2.6)_{1}$ it follows that

$$
u_{k, l}(\mathbf{x})=i \sum_{n_{j}=0}^{ \pm \infty} \hat{u}_{k}(\xi) \xi_{l} e^{i \xi \cdot \mathbf{x}}
$$

where a prime on $\sum$ indicates that in the summation, $n=\left(n_{j} n_{j}\right)^{1 / 2}=0$ is excluded. ${ }^{3}$ From (2.7) and $(2.6)_{2}$, one now obtains upon substitution into (2.5)

$$
-C_{r j k l} \hat{u}_{k} \xi_{l} \xi_{j}=i C_{r j k l} \hat{\hat{k}}_{k l}^{*} \xi_{j}
$$

which can be solved for $\hat{u}_{k}$ to yield

where

$$
\hat{u}_{k}(\xi)=-i C_{r j p l} \hat{\varepsilon}_{p l}^{*} \xi_{j} N_{r k}(\xi),
$$

$$
N_{r k}(\xi)=\left[\delta_{r k}(\lambda+2 \mu) \xi^{2}-(\lambda+\mu) \xi_{r} \xi_{k}\right] /(\lambda+2 \mu) \mu \xi^{4}, \quad \xi^{2}=\xi_{j} \xi_{j} \neq 0 .
$$

\footnotetext{
${ }^{1}$ Even for ellipsoidal cavities, $\varepsilon_{i j}^{*}$ will not be a constant in the present case.

${ }^{2}$ Here and in the sequel $i$ is used to indicate $\sqrt{ }-1$, although it is occasionally also used as a subscript index. The context makes the usage clear.

${ }^{3}$ From (2.6), it is observed that the constant term associated with $n=0$ is automatically excluded (by differentiation) in (2.7), and hence this term does not enter the integral equations (2.11) and (2.13).
} 
Upon substitution from (2.8) into (2.7), one arrives at

$$
u_{k, l}(\mathbf{x})=\sum_{n_{j}=0}^{ \pm \infty} C_{p q r s} \hat{\varepsilon}_{r s}^{*}(\xi) \xi_{q} \xi_{l} N_{k p}(\xi) e^{i \xi \cdot \mathbf{x}}
$$

Finally, by direct substitution into (2.4), one deduces the following integral equation for the unknown transformation strain $\varepsilon_{k l}^{*}$ :

$$
-\varepsilon_{k l}^{0}=\sum_{n_{j}=n}^{ \pm \infty} F_{k l r s}(\xi) \frac{1}{\Lambda_{1} \Lambda_{2} \Lambda_{3}} \int_{\Omega} \varepsilon_{r s}^{*}\left(\mathbf{x}^{\prime}\right) e^{i \xi \cdot\left(\mathbf{x}-\mathbf{x}^{\prime}\right)} d \mathbf{x}^{\prime}-\varepsilon_{k l}^{*}(\mathbf{x})
$$

where we have set

$$
\begin{aligned}
& F_{k l r s}(\xi)= \frac{1}{2} C_{p q r s} \xi_{q}\left[\xi_{l} N_{k p}(\xi)+\xi_{k} N_{l p}(\xi)\right] \\
&= \frac{1}{2 n^{2}}\left\{\left[n_{s} \delta_{k r}+n_{r} \delta_{k s}+\left(\frac{\lambda}{\lambda+2 \mu}\right) n_{k} \delta_{r s}\right] n_{l}+\left[n_{s} \delta_{l n}+n_{r} \delta_{l s}+\left(\frac{\lambda}{\lambda+2 \mu}\right) n_{l} \delta_{r s}\right] n_{k}\right\} \\
&-\frac{2}{n^{4}}\left(\frac{\lambda+\mu}{\lambda+2 \mu}\right) n_{k} n_{l} n_{r} n_{s}, \\
& \xi_{j}= 2 \pi n_{j} / \Lambda_{j}(\text { no sum on } j), \\
& n^{2}=n_{l} n_{l} \neq 0, \quad k, l, r, s=1,2,3, \quad n_{l}=0, \pm 1, \ldots,
\end{aligned}
$$

and where, in arriving at (2.11), we have also used the identity $\sigma_{i j}^{0}=C_{i j k l} \varepsilon_{k l}^{0}$.

For the calculation of the effective elastic moduli only the average value of the transformation strain taken over the void volume is required. Integrating both sides of (2.11) over the void volume and dividing the results by the void volume $\Omega$, we obtain

$$
\begin{aligned}
\bar{\varepsilon}_{k l}^{*} & =\frac{1}{\Omega} \int_{\Omega} \varepsilon_{k l}^{*}(\mathbf{x}) d \mathbf{x} \\
& =\varepsilon_{k l}^{0}+\frac{1}{\Omega \Lambda_{1} \Lambda_{2} \Lambda_{3}} \sum_{n_{j}=0}^{ \pm \infty} F_{k l r s}(\xi) g_{0}(\xi) \int_{\Omega} \varepsilon_{r s}^{*}\left(\mathbf{x}^{\prime}\right) e^{-i \xi \cdot \mathbf{x}^{\prime}} d \mathbf{x}^{\prime},
\end{aligned}
$$

where

$$
g_{0}(\xi)=\int_{\Omega} e^{i \xi \cdot \mathbf{x}} d \mathbf{x}
$$

3. Solution methods. We shall now consider several solution methods for Eqs. (2.11) and (2.13). To render our results more explicit, we shall confine attention first to the case where each unit cell contains only one spherical void of radius $R$, with the center located at the center of the unit cell which we choose as the origin of the coordinate system. Then we generalize our results for application to cases where the unit cell may include one or several voids of possibly dissimilar geometries, e.g. one spherical void and one cylindrical void. Note that in the case of equally spaced spherical voids, we have a cubic unit cell. Hence we set $\Lambda_{1}=\Lambda_{2}=\Lambda_{3}=\Lambda$. 
3.1 Periodically distributed spherical voids. In this case we need to calculate the integral of $e^{ \pm i \xi \cdot x}$ over the spherical region $\Omega$. Using spherical coordinates, we obtain

$$
\begin{array}{rlrl}
g_{0}(\xi) & =\int_{\Omega} e^{ \pm i \xi \cdot x} d \mathbf{x}=\frac{4 \pi}{\xi} \int_{0}^{R} r \sin \xi r d r, \\
& =\frac{4 \pi}{\xi^{3}}(\sin \xi R-\xi R \cos \xi R) & & \text { if } \xi \neq 0 \\
& =\frac{4 \pi}{3} R^{3} & \text { if } \xi=0, \xi^{2}=\xi \cdot \xi .
\end{array}
$$

With the aid of this result, we first examine the convergence character of the infinite series ${ }^{4}$ in (2.13). To this end we observe that the function $F_{k l r s}(\xi)$ remains finite as $n_{j}$ (and hence $\xi_{j}$ ) takes on large values. On the other hand, both the integral and the term $g_{0}(\xi)$ in this series go to zero like $1 / n^{2}$ as $n_{j} \rightarrow \pm \infty$, and hence the infinite series converges absolutely, its terms going to zero like $1 / n^{4}$ as $n_{j} \rightarrow \pm \infty$. To see this, we estimate the integral as follows. The transformation strain is bounded, $\left|\varepsilon_{r s}^{*}\right| \leq M$ in $\Omega$, where $M$ is a positive finite constant. Then

$$
\begin{aligned}
\left|\int_{\Omega} \varepsilon_{r s}^{*}\left(\mathbf{x}^{\prime}\right) e^{-i \xi \cdot \mathbf{x}^{\prime}} d \mathbf{x}^{\prime}\right| & \leq M\left|\int_{\Omega} e^{-i \xi \cdot x^{\prime}} d \mathbf{x}^{\prime}\right| \\
& =\frac{4 \pi M}{\xi^{3}}|\sin \xi R-\xi R \cos \xi R| \\
& \leq 4 \pi M\left(1 / \xi^{3}+R / \xi^{2}\right), \quad \xi^{2}=\xi \cdot \xi \neq 0,
\end{aligned}
$$

which shows that the terms of the infinite series in (2.13) are of the order of $1 / \xi^{4}$ for large values of $\xi$.

We shall now examine several methods for solving Eqs. (2.11) and (2.13), starting with the simplest approximation which utilizes Eq. (2.13).

3.2 Simplest approximation. The structure of Eq. (2.13) suggests a simple method for approximating $\bar{\varepsilon}_{k l}^{*}$. It is obtained if we estimate the integral in the right-hand side as follows:

$$
\int_{\Omega} \varepsilon_{r s}^{*}\left(\mathbf{x}^{\prime}\right) e^{-i \xi \cdot \mathbf{x}^{\prime}} d \mathbf{x}^{\prime} \approx \bar{\varepsilon}_{r s}^{*} \int_{\Omega} e^{-i \xi \cdot \mathbf{x}^{\prime}} d \mathbf{x}^{\prime}=\bar{\varepsilon}_{r s}^{*} g_{0}(\xi)
$$

Then (2.13) yields

$$
\left[\delta_{k r} \delta_{l s}-\frac{1}{\Omega \Lambda^{3}} \sum_{n_{j}=0}^{ \pm \infty} F_{k l r s}(\xi) g_{0}^{2}(\xi)\right] \bar{\varepsilon}_{r s}^{*}=\varepsilon_{k l}^{0},
$$

from which $\bar{\varepsilon}_{r s}^{*}$ can be calculated by matrix inversion.

It should be noted that the approximate solution (3.4) does indeed take into account void interaction effects as well as the influence of void geometry. In this solution the quantity $g_{0}(\xi)$ reflects the void geometry, whereas the value of the infinite series accounts

\footnotetext{
${ }^{4}$ We shall not attempt to solve (2.11) directly, and therefore we do not discuss the convergence of the infinite series involved.
} 
for the void interaction. Therefore the corresponding results are expected to be quite accurate, as, in fact, they are; this will be shown by means of examples later on.

Examples. As an illustrative example, we apply Eq. (3.4) to the case where $\varepsilon_{k l}^{0}=\varepsilon^{0} \delta_{k l}$, i.e. uniform dilatation. Hence, we take $\bar{\varepsilon}_{k l}^{*}=\bar{\varepsilon}^{*} \delta_{k l}$, and observe that (3.4) yields

$$
\begin{aligned}
& \frac{\bar{\varepsilon}^{*}}{\varepsilon^{0}}=\left[1-\frac{16 \pi^{2}}{3 \Omega \Lambda^{3}} \sum_{n_{j}=0}^{ \pm \infty} \frac{(\sin \xi R-\xi R \cos \xi R)^{2}}{\xi^{6}}\right]^{-1}, \\
& \xi^{2}=\xi_{j} \xi_{j}, \quad \xi_{j}=2 \pi n_{j} / \Lambda, \quad n_{j}= \pm 0, \pm 1, \ldots .
\end{aligned}
$$

We note that the infinite series in (3.5) converges rapidly. In Sec. 5 the accuracy and convergence rate of $(3.5)$ are illustrated.

As a second example, consider a simple shearing where $\varepsilon_{12}^{0} \neq 0$ and $\bar{\varepsilon}_{12}^{*} \neq 0$, while all other components are zero. Eq. (3.4) then yields

$$
\frac{\bar{\varepsilon}_{12}^{*}}{\varepsilon_{12}^{0}}=\left[1-\frac{32 \pi^{2}}{\Omega \Lambda^{3}} \sum_{n_{j}=0}^{ \pm \infty}\left|\frac{\left(\xi_{1}^{2}+\xi_{2}^{2}\right)}{2 \xi^{2}}-\left(\frac{\lambda+\mu}{\lambda+2 \mu}\right) \frac{\xi_{1}^{2} \xi_{2}^{2} \mid}{\xi^{4}}\right| \frac{(\sin \xi R-\xi R \cos \xi R)^{2}}{\xi^{6}}\right]^{-1} .
$$

In Sec. 5, this and related results are illustrated numerically.

We note that simple shear may be produced by setting $\varepsilon_{11}^{0}=\varepsilon^{0}, \varepsilon_{22}^{0}=-\varepsilon^{0}$, and $\bar{\varepsilon}_{11}^{*}=\bar{\varepsilon}^{*}, \bar{\varepsilon}_{22}^{*}=-\bar{\varepsilon}^{*}$, with all other components being zero. Then Eq. (3.4) yields

$$
\begin{aligned}
\frac{\bar{\varepsilon}^{*}}{\varepsilon^{0}} & =\left[1-\frac{1}{\Omega \Lambda^{3}} \sum_{n_{j}=0}^{ \pm \infty}\left\{F_{1111}(\xi)-F_{1122}(\xi)\right\} g_{0}^{2}(\xi)\right]^{-1} \\
& \left.=\left[1-\frac{2}{\Omega \Lambda^{3}} \sum_{n_{j}=0}^{ \pm \infty}\left(\frac{\xi_{1}}{\xi}\right)^{2} \mid 1-\frac{\lambda+\mu}{\lambda+2 \mu}\left(\left(\frac{\xi_{1}}{\xi}\right)^{2}-\left(\frac{\xi_{2}}{\xi}\right)^{2}\right)\right\} g_{0}^{2}(\xi)\right]^{-1} .
\end{aligned}
$$

The values obtained from this equation are discussed in Sec. 5, where it is shown that, (3.6) and (3.7) do not yield identical results, as has also been observed by Christoffersen [10], using a different approach. ${ }^{5}$

3.3 Polynomial approximation. We shall now consider more accurate estimates for the transformation strain, using Eq. (2.11). To this end we approximate the transformation strain by a polynomial with unknown coefficients which are then calculated with the aid of (2.11) and either a Galerkin method or a variational approach. In the present context, the former yields a more effective computational tool, whereas the latter involves series which may not even converge.

For illustration we consider the case where the unit cells are cubes of dimension $\Lambda$ and contain a centrally located spherical void of radius $R$. Then we estimate the transformation strain by

$$
\varepsilon_{k l}^{*}(\mathbf{x})=C_{k l}^{\alpha}|\mathbf{x} / R|^{\alpha} \quad(\text { sum on } \alpha), \quad \alpha=0,1,2, \ldots, K,
$$

substitute this into (2.11), and carry out the required integration. For the spherical void, all the integrals involved can be reduced to

$$
g_{\alpha}(\xi)=\int_{\Omega} e^{-i \xi \cdot \mathbf{x}}|\mathbf{x}|^{\alpha} d \mathbf{x}=\frac{4 \pi}{\xi} \int_{0}^{R} r^{1+\alpha} \sin \xi r d r
$$

${ }^{5}$ This is because the simple cubic arrangement of voids does not result in isotropy. 
where the last expression is obtained by employing spherical coordinates. For $\alpha=0,(3.1)$ yields the required integral. For other values of $\alpha$, we use the recursion relation

$$
\int_{0}^{R} r^{1+\alpha} \sin \xi r d r=-\frac{1}{\xi} R^{1+\alpha} \cos \xi R+\frac{1+\alpha}{\xi^{2}} R^{\alpha} \sin \xi R-\frac{(1+\alpha) \alpha}{\xi^{2}} \int_{0}^{R} r^{\alpha-1} \sin \xi r d r .
$$

In Appendix A explicit expressions for $g_{\alpha}$ are listed.

With the aid of (3.8) and (3.9), the integral equation (2.11) is reduced to

$$
\varepsilon_{k l}^{0}=\left[\delta_{k r} \delta_{l s}\left|\frac{\mathbf{x}}{R}\right|^{\alpha}-\frac{1}{\Lambda^{3} R^{\alpha}} \sum_{n_{j}=0}^{ \pm \infty} F_{k l r s}(\xi) g_{\alpha}(\xi) e^{i \xi \cdot \mathbf{x}}\right] C_{r s}^{\alpha}, \quad \alpha=0,1, \ldots, K .
$$

To calculate the unknown coefficients $C_{r s}^{x}$ from this equation, we may use a Galerkin method, as discussed below.

Galerkin method. To obtain a system of linear equations for the unknown coefficients $C_{r s}^{\alpha}$, we multiply both sides of Eq. (3.11) by $|\mathbf{x} / R|^{\beta}$ for $\beta=0,1, \ldots, K$, and integrate the resulting equations over the void volume $\Omega$ in order to arrive at the following system of linear equations:

$$
\left[\delta_{k r} \delta_{l s} \frac{4 \pi R^{3}}{\alpha+\beta+3}-\frac{1}{\Lambda^{3} R^{\alpha+\beta}} \sum_{n j=0}^{ \pm \infty} F_{k l r s}(\xi) g_{\alpha}(\xi) g_{\beta}(\xi)\right] C_{r s}^{\alpha}=\frac{4 \pi R^{3}}{\beta+3} \varepsilon_{k l}^{0}
$$

As seen from Appendix A, the quantity $g_{\alpha}$ is of the order of $1 / \xi^{2}$ for large $\xi$. Therefore, the infinite series in (3.12) converges like $1 / \xi^{4}$.

We note that for $\alpha=\beta=0$, Eq. (3.12) reduces to (3.4) if we identify $C_{r s}^{0}$ with $\bar{\varepsilon}_{r s}^{*}$. Hence the coefficients $C_{r s}^{\alpha}$ for $\alpha=1,2, \ldots, K$ represent the deviation of the transformation strain from uniformity.

Since only the average value of the transformation strain enters the calculation of the effective elastic moduli, from (3.8) we have

$$
\bar{\varepsilon}_{k l}^{*}=C_{k l}^{\alpha} \frac{1}{\Omega} \int_{\Omega}\left|\frac{\mathbf{x}}{R}\right|^{\alpha} d \mathbf{x}=C^{0}+\frac{3}{4} C^{1}+\frac{3}{5} C^{2}+\cdots .
$$

Using a three-term approximation, i.e. $\alpha, \beta=0,1,2$, we have calculated the results obtained from the system of equations (3.12), which correspond to uniform dilatation and simple shear. These and related numerical results are presented and discussed in Sec. 5 .

Variational method. The calculation of the unknown coefficients in the polynomial approximation (3.8) may be based on a variational approach. Although this may sound more appealing, it leads to infinite series which may not be convergent. Here we shall obtain the corresponding equations.

There are various ways by which a relevant variational statement can be constructed. The simplest seems to be to use the virtual work method. To this end we observe from Eq. (2.3) that the total stress must vanish within the voids in a typical cell. With $\delta u_{j}$ representing a smooth virtual displacement and $\delta u_{j, i}$ the corresponding gradient, we 
multiply both sides of $(2.3)$ by the virtual displacement gradient $\delta u_{j, i}$, sum over $i$ and $j$, integrate the results over $\Omega$, and arrive at

$$
\int_{\Omega} C_{i j k l}\left(u_{k, l}^{0}+u_{k, l}(\mathbf{x})-\varepsilon_{k l}^{*}(\mathbf{x})\right) \delta u_{i, j}(\mathbf{x}) d \mathbf{x}=0 .
$$

We now approximate the transformation strain by (3.8) and substitute into (2.10) to obtain

$$
u_{i, j}(\mathbf{x})=\left[\frac{1}{\Lambda^{3}} \sum_{n_{j}=0}^{ \pm \infty} F_{i j m n}(\xi) g_{\alpha}(\xi) e^{i \xi \cdot \mathbf{x}}\right] C_{m n}^{\alpha} .
$$

Then $\delta u_{i, j}$ is obtained from (3.12), if we replace $C_{m n}^{\alpha}$ by $\delta C_{m n}^{\alpha}$. Now upon substitution into (3.14), we arrive at

$$
\begin{array}{r}
{\left[\frac{1}{\Lambda^{3}} C_{i j k l} \sum_{n_{j}=0}^{ \pm \infty} \sum_{m_{j}=0}^{ \pm \infty} F_{k l r s}(\xi) F_{i j m n}(\boldsymbol{\eta}) g_{0}(\xi+\eta) g_{\alpha}(\xi) g_{\beta}(\boldsymbol{\eta})-\right.} \\
\left.-C_{i j r s} \sum_{n_{j}=0}^{ \pm \infty} F_{i j m n}(\xi) g_{\alpha}(\xi) g_{\beta}(\xi)\right] C_{r s}^{\alpha} \\
=\sigma_{i j}^{0} \sum_{n_{j}=0}^{ \pm \infty} F_{i j m n}(\xi) g_{0}(\xi) g_{\beta}(\xi),
\end{array}
$$

where we have set equal to zero the coefficients of $\delta C_{i j}^{\beta}$ and where $\sigma_{i j}^{0}=C_{i j k l} \varepsilon_{k l}^{0}$. In this system of linear equations the double-triple infinite series in the left-hand side of (3.16) is a source of considerable computational difficulties, and indeed may not be convergent. This makes the method ineffective.

3.4 A complete solution method. Since the kernel of the integral in (2.11) is separable, this equation may be reduced to a system of linear algebraic equations for the unknown coefficients

$$
E_{r s}(\xi)=\int_{\Omega} \varepsilon_{r s}^{*}\left(\mathbf{x}^{\prime}\right) e^{-i \xi \cdot \mathbf{x}^{\prime}} d \mathbf{x}^{\prime}
$$

To this end we multiply both sides of (2.11) by $e^{-i \boldsymbol{\eta} \cdot \mathbf{x}}$, integrate the results over $\Omega$ and, rearranging terms, arrive at

$$
-g_{0}(\boldsymbol{\eta}) \varepsilon_{k l}^{0}=-E_{k l}(\boldsymbol{\eta})+\frac{1}{\Lambda^{3}} \sum_{n_{j}=0}^{ \pm \infty} F_{k l r s}(\xi) g_{0}(\boldsymbol{\eta}-\xi) E_{r s}(\xi) .
$$

This is an infinite system of linear equations for the unknown coefficients $E_{r s}(\xi)$. For each fixed $\boldsymbol{\eta}$ the infinite series in the right-hand side converges better than $1 / \xi^{4}$. This can be seen from (3.1) and the estimate (3.2). Moreover, these unknown coefficients go to zero with increasing $\xi$ like $1 / \xi^{2}$. Hence, in actual calculations only a finite number of equations and the same finite number of terms for the infinite series may be employed. In this manner, the final solution yields

$$
\varepsilon_{k l}^{*}(\mathbf{x}) \approx \varepsilon_{k l}^{0}+\sum_{n_{j}=0}^{ \pm \infty} F_{k l r s}(\xi) E_{r s}(\xi) e^{i \xi \cdot \mathbf{x}}
$$

where the infinite series is truncated after $n_{j}= \pm N$, the corresponding number of linear equations in (3.18) then being $(2 N+1)^{3}$.

We note that in the matrix representation of the right-hand side of (3.18) the matrix of the coefficients of the unknowns is strongly dominated by diagonal elements, and the 
off-diagonal elements diminish like $1 /|\boldsymbol{\eta}-\xi|^{2}$. Hence, one may not need to include too many terms in order to obtain reasonable estimates. Moreover, for the calculation of the effective elastic moduli, we need to obtain only the most dominant terms in (3.18), namely

$$
E_{r s}(\mathbf{0})=\int_{\Omega} \varepsilon_{r s}^{*}\left(\mathbf{x}^{\prime}\right) d \mathbf{x}^{\prime}
$$

3.5 Generalization. In all the equations presented above for various solution schemes, the only quantity which relates to the void geometry is $g_{\alpha}(\xi)$. Therefore the basic solution schemes can be applied to any porous elastic medium which consists of a collection of cubical (or parallelepiped) unit cells, each containing the same number of voids with the same geometrical structure. For example, if one considers a rectangular void of dimensions $a_{i}, i=1,2,3$, in each unit cell, then the function $g_{0}(\xi)$ becomes

$$
\begin{aligned}
g_{0}(\xi) & =\frac{8}{\xi_{1} \xi_{2} \xi_{3}} \sin a_{1} \xi_{1} \sin a_{2} \xi_{2} \sin a_{3} \xi_{3} \quad \text { if } \xi \neq 0 \\
& =a_{1} a_{2} a_{3} \quad \text { if } \xi=0 .
\end{aligned}
$$

In a similar manner, one can obtain results for ellipsoidal, cylindrical, and other void geometries. Moreover, if there are several voids with different geometries, then we simply replace the corresponding $g_{\alpha}$ functions by the sum of the corresponding quantities.

4. Effective elastic moduli. We shall estimate the effective elastic moduli of a porous elastic medium containing periodically distributed voids by considering the strain energy. To this end let the body occupy the region $\bar{D}$ with the boundary $S$ on which (selfequilibrating) tractions $T_{i}$ are prescribed. Using the notation of Sec. 2, the total potential energy is

$$
W=\frac{1}{2} \int_{D}\left(\sigma_{i j}^{0}+\sigma_{i j}\right)\left(u_{i, j}^{0}+u_{i, j}\right) d \mathbf{x}-\int_{S} T_{i}\left(u_{i}^{0}+u_{i}\right) d S,
$$

where quantities denoted by superscript zero are the fields which would be induced if the solid were homogeneous and voidless and the corresponding quantities without superscript zero are the perturbations produced because of the existence of voids. If we denote the total potential energy of the body in the absence of voids but subjected to the same boundary conditions by $W_{0}$, then the change in the potential energy $\Delta W$ is given by

$$
\begin{aligned}
\Delta W & =W-W_{0}=\frac{1}{2} \int_{\bar{D}}\left[\sigma_{i j}^{0} u_{i, j}+\sigma_{i j}\left(u_{i, j}^{0}+u_{i, j}\right)\right] d \mathbf{x}-\int_{S} T_{i} u_{i} d S \\
& =\int_{\bar{D}}\left[\sigma_{i j}^{0} u_{i, j}-\frac{1}{2} \sigma_{i j}^{0} \varepsilon_{i j}^{*}+\frac{1}{2} \sigma_{i j} u_{i, j}\right] d \mathbf{x}-\int_{S} T_{i} u_{i} d S \\
& =-\frac{1}{2} \int_{\bar{\Omega}} \sigma_{i j}^{0} \varepsilon_{i j}^{*} d \mathbf{x},
\end{aligned}
$$

where we have used Hooke's law, the Gauss theorem, and the fact that both $\sigma_{i j}^{0}$ and $\sigma_{i j}$ are stress fields in equilibrium with zero body forces. Moreover, we have noted that the transformation strain $\varepsilon_{i j}^{*}$ is zero in $\bar{D}-\bar{\Omega}$, where $\bar{\Omega}$ is the region occupied by all voids contained in the total domain $\bar{D}$. 
Denote by $C_{i j k l}^{*}$ the overall effective elastic modulus tensor of the porous body, and let $D_{i j k l}^{*}$ be the corresponding elastic compliance; the elastic compliance of the matrix will be denoted by $D_{i j k l}$. By definition, the total elastic energy of the porous body is $\frac{1}{2} D_{i j k l}^{*} \sigma_{i j}^{0} \sigma_{k l}^{0}$, which, in view of (4.2), can be expressed as

$$
D_{i j k l}^{*} \sigma_{i j}^{0} \sigma_{k l}^{0}=D_{i j k l} \sigma_{i j}^{0} \sigma_{k l}^{0}+\frac{1}{\bar{D}} \int_{\bar{\Omega}} \sigma_{i j}^{0} \varepsilon_{i j}^{*} d \mathbf{x}
$$

and since, in the context of the present work, $\sigma_{i j}^{0}$ is constant, we obtain

$$
D_{i j k l}^{*} \sigma_{i j}^{0} \sigma_{k l}^{0}=D_{i j k l} \sigma_{i j}^{0} \sigma_{k l}^{0}+\frac{\sigma_{i j}^{0} \Omega \bar{\varepsilon}_{i j}^{*}}{\Lambda_{1} \Lambda_{2} \Lambda_{3}}
$$

where $\bar{\varepsilon}_{i j}^{*}$ is the average value of the transformation strain over $\Omega$, defined in Eq. (2.13); note that $\Omega \bar{\varepsilon}_{i j}^{*}=E_{i j}(\mathbf{0})$, as seen from (3.17).

We now decompose the applied stress into the dilatational $\sigma^{0}$ and distortional $\tau_{i j}^{0}$ parts as

$$
\sigma_{i j}^{0}=\tau_{i j}^{0}+\frac{1}{3} \sigma^{0} \delta_{i j},
$$

and under the assumption of overall isotropy ${ }^{6}$ obtain from (4.4)

$$
\frac{\sigma^{02}}{9 K^{*}}+\frac{\tau_{i j}^{0} \tau_{i j}^{0}}{2 \mu^{*}}=\frac{\sigma^{02}}{9 K}+\frac{\tau_{i j}^{0} \tau_{i j}^{0}}{2 \mu}+\frac{\Omega\left(\tau_{i j}^{0} \bar{\varepsilon}_{i j}^{*}+\frac{1}{3} \sigma^{0} \bar{\varepsilon}_{k k}^{*}\right)}{\Lambda^{3}},
$$

where $K^{*}$ and $\mu^{*}$ are, respectively, the overall bulk and shear moduli of the porous elastic body, $K$ and $\mu$ those of the matrix, and where $\Omega=4 \pi R^{3} / 3$ and $\Lambda$ is the void spacing. From Eq. (4.6) we can calculate the effective moduli from the knowledge of $\bar{\varepsilon}_{i j}^{*}$.

Examples. First consider a uniform dilatational stress field $\sigma_{i j}^{0}=\frac{1}{3} \sigma^{0} \delta_{i j}$, and from (4.6) obtain

$$
\frac{K}{K^{*}}=1+\left(3 K f / \sigma^{0}\right) \bar{\varepsilon}_{k k}^{*}=1+f\left(\bar{\varepsilon}_{k k}^{*} / \varepsilon^{0}\right), \quad \varepsilon^{0}=\sigma^{0} / 3 K,
$$

where $f=4 \pi R^{3} / 3 \Lambda^{3}$ is the void volume fraction.

Next, consider a uniform distortional field, $\tau_{12}^{0}=\tau_{21}^{0}=\tau^{0}$, with all other components of $\tau_{i j}^{0}$ being zero. Then (4.6) yields

$$
\frac{\mu}{\mu^{*}}=1+\left(2 \mu f / \tau^{0}\right) \bar{\varepsilon}_{12}^{*}=1+f\left(\bar{\varepsilon}_{12}^{*} / \varepsilon_{12}^{0}\right), \quad \varepsilon_{12}^{0}=\tau^{0} / 2 \mu .
$$

On the other hand, if the overall shear is produced by $\sigma_{11}^{0}=\sigma^{0}$ and $\sigma_{22}^{0}=-\sigma^{0}$, with all other components being zero, we obtain

$$
\frac{\mu}{\mu^{*}}=1+\left(2 \mu f / \sigma^{0}\right) \bar{\varepsilon}^{*}=1+f\left(\bar{\varepsilon}^{*} / \varepsilon^{0}\right), \quad \varepsilon^{0}=\sigma^{0} / 2 \mu .
$$

Note that $\bar{\varepsilon}_{k k}^{*} / \varepsilon^{0}, \bar{\varepsilon}_{12}^{*} / \varepsilon_{12}^{0}$, and $\bar{\varepsilon}^{*} / \varepsilon^{0}$ are given in the preceding section.

\footnotetext{
${ }^{6}$ Even when the matrix is isotropic, the void's geometry and spacing may result in an overall anisotropy; however, the simple cubic arrangement of spherical voids does not result in isotropy.
} 
TABLE 1. Estimates of the effective bulk modulus: the simplest approximation, Eqs. (3.5) and (4.7).

\begin{tabular}{|c|c|c|c|c|c|c|c|c|c|c|c|c|}
\hline \multirow{2}{*}{$\begin{array}{l}\text { Void volume } \\
\text { fraction, } f \\
(\%)\end{array}$} & \multicolumn{2}{|c|}{$N=1$} & \multicolumn{2}{|c|}{$N=3$} & \multicolumn{2}{|c|}{$N=4$} & \multicolumn{2}{|c|}{$N=10$} & \multicolumn{2}{|c|}{$N=25$} & \multicolumn{2}{|c|}{$N=45$} \\
\hline & $K^{*} / K$ & $\bar{\varepsilon}^{*} / \varepsilon^{0}$ & $K^{*} / K$ & $\bar{\varepsilon}^{*} / \varepsilon^{0}$ & $K^{*} / K$ & $\bar{\varepsilon}^{*} / \varepsilon^{0}$ & $K^{*} / K$ & $\bar{\varepsilon}^{*} / \varepsilon^{0}$ & $K^{*} / K$ & $\bar{\varepsilon}^{*} / \varepsilon^{0}$ & $K^{*} / K$ & $\bar{\varepsilon}^{*} / \varepsilon^{0}$ \\
\hline 5 & 0.930 & 1.511 & 0.911 & 1.950 & 0.907 & 2.057 & 0.899 & 2.250 & 0.895 & 2.351 & 0.894 & 2.384 \\
\hline 10 & 0.856 & 1.681 & 0.838 & 1.932 & 0.834 & 1.983 & 0.824 & 2.134 & 0.819 & 2.205 & 0.818 & 2.228 \\
\hline 15 & 0.801 & 1.659 & 0.783 & 1.844 & 0.778 & 1.904 & 0.768 & 2.015 & 0.763 & 2.070 & 0.762 & 2.088 \\
\hline 20 & 0.760 & 1.577 & 0.740 & 1.761 & 0.734 & 1.809 & 0.724 & 1.904 & 0.720 & 1.948 & 0.718 & 1.963 \\
\hline 25 & 0.728 & 1.493 & 0.703 & 1.689 & 0.699 & 1.721 & 0.689 & 1.803 & 0.685 & 1.840 & 0.684 & 1.852 \\
\hline 30 & 0.701 & 1.421 & 0.673 & 1.618 & 0.670 & 1.645 & 0.661 & 1.711 & 0.657 & 1.742 & 0.656 & 1.752 \\
\hline
\end{tabular}

5. Discussion and numerical results. For an elastic body containing periodically distributed voids of arbitrary geometry, several computational schemes were presented in the preceding section by means of which the effective elastic moduli and the stress and strain fields can be estimated. Here we shall examine and illustrate in detail the convergence behavior of the infinite series involved give several numerical examples, and compare the resulting estimates of the effective elastic moduli with those reported by others using different methods.

Consider first the infinite series in Eq. (3.5). The individual terms in this series depend only on the magnitude of the vector $\xi_{j}=2 \pi n_{j} / \Lambda, n_{j}= \pm 0,+1, \ldots, \pm N$, if the series is truncated at $n_{j}= \pm(N+1)$. The dominant term in this series is $\cos ^{2} \xi R / \xi^{4}$. Hence the total truncation error is less than $A / \xi(N+1)$, where $A$ is a positive constant. In view of this, we need only to include the term associated with $n_{j}$ for which the magnitude of the vector $\xi_{j}$ is less than or equal to $2 \pi N / \Lambda$. Similar comments apply to the infinite series involved in Eqs. (3.6), (3.7), and (3.12).

We have calculated the right-hand sides of Eqs. (3.5), (3.6) and (3.7) for different values of $N$ in order to examine the convergence of the involved series.

The calculations pertaining to the estimate of the bulk modulus, using the simplest approximation, Eq. (3.5), together with Eq. (4.7), are shown in Table 1 for the values of $N$ indicated and the void volume fraction $f=4 \pi R^{3} / 3 \Lambda^{3}$. As is seen, convergence is reasonable. In addition to this, we have also obtained results using the complete solution method of Sec. 3.4. This involves considerable computational effort, and is used to confirm the estimates presented in Table 1. For example, for $N=4$ and $f=30 \%$, the

TABLE 2. Estimates of the effective bulk modulus: Galerkin approximations, $N=4$.

\begin{tabular}{|c|c|c|c|c|c|c|c|c|c|}
\hline \multirow{2}{*}{$\begin{array}{l}\text { Void volume } \\
\text { fraction, } f \\
(\%)\end{array}$} & \multicolumn{4}{|c|}{ Two-term approximation } & \multicolumn{5}{|c|}{ Three-term approximation } \\
\hline & $K^{*} / K$ & $\bar{\varepsilon}^{*} / \varepsilon^{0}$ & $C^{0}$ & $C^{1}$ & $K^{*} / K$ & $\bar{\varepsilon}^{*} / \varepsilon^{0}$ & $C^{0}$ & $C^{1}$ & $C^{2}$ \\
\hline 5 & 0.904 & 2.131 & 3.635 & -2.006 & 0.902 & 2.169 & 1.120 & 5.948 & -5.687 \\
\hline 10 & 0.831 & 2.033 & 3.287 & -1.672 & 0.829 & 2.065 & 0.766 & 6.414 & -5.853 \\
\hline 15 & 0.775 & 1.938 & 2.991 & -1.403 & 0.773 & 1.961 & 0.847 & 5.491 & -5.007 \\
\hline 20 & 0.731 & 1.836 & 2.791 & -1.273 & 0.729 & 1.857 & 0.757 & 5.272 & -4.758 \\
\hline 25 & 0.696 & 1.743 & 2.598 & -1.140 & 0.694 & 1.760 & 0.734 & 4.871 & -4.378 \\
\hline 30 & 0.667 & 1.662 & 2.411 & -0.999 & 0.665 & 1.676 & 0.723 & 4.450 & -3.975 \\
\hline
\end{tabular}


TABLE 3. Estimates of the effective shear modulus: the simplest approximation, Eqs. (3.6) and (4.8).

\begin{tabular}{ccccc}
\hline $\begin{array}{c}\text { Void volume } \\
\text { fraction, } f \\
(\%)\end{array}$ & $N=4$ & $N=10$ & $N=25$ & $N=45$ \\
\hline 5 & 0.941 & 0.917 & 0.915 & 0.914 \\
10 & 0.888 & 0.847 & 0.844 & 0.843 \\
15 & 0.841 & 0.789 & 0.786 & 0.785 \\
20 & 0.800 & 0.742 & 0.738 & 0.737 \\
25 & 0.763 & 0.702 & 0.699 & 0.698 \\
30 & 0.731 & 0.670 & 0.667 & 0.666 \\
\hline
\end{tabular}

simplest approximation gives $K^{*} / K=0.670$, whereas our complete solution gives $K^{*} / K=0.664$.

The simplest approximation is actually a one-term Galerkin estimate, as is seen by comparing Eq. (3.5) with (3.12) and (3.13). In Table 2 the estimates for the effective bulk modulus obtained by two- and three-term Galerkin approximations, are summarized for $N=4$. As is seen, again the simplest approximation reported in Table 1 appears quite adequate.

The estimates of the effective shear modulus obtained by the simplest approximation, Eqs. (3.6) and (4.8), are presented in Table 3 for various values of $N$. Here the rate of convergence of the infinite series involved follows essentially the same pattern as that displayed in Table 1. In Table 4 we have compared the results of Eqs. (3.6) and (4.8) with those of (3.7) and (4.9) for $N=4$. The corresponding estimates by the three-term Galerkin approximation are given in Table 5. Finally, Table 6 summarizes for $N=4$ the estimates obtained by means of the complete solution method of Sec. 3.4.

An examination of these numerical results reveals that the basic method is indeed very effective and that, in all cases, the simplest approximation provides adequate estimates.

TABLE 4. Estimates of the effective shear modulus: the simplest approximation, $N=4$.

\begin{tabular}{cccccc}
\hline $\begin{array}{c}\text { Void volume } \\
\text { fraction, } f \\
(\%)\end{array}$ & \multicolumn{2}{l}{ Eqs. (3.6) and $(4.8) \dagger$} & & \multicolumn{2}{c}{ Eqs. (3.7) and $(4.9)_{+}^{+}$} \\
\cline { 2 - 3 } \cline { 5 - 6 } & $\mu^{*} / \mu$ & $\bar{\varepsilon}_{12}^{*} / \varepsilon_{12}^{0}$ & & $\mu^{*} / \mu$ & $\bar{\varepsilon}^{*} / \varepsilon^{0}$ \\
\hline 5 & 0.941 & 1.263 & & 0.950 & 1.064 \\
10 & 0.888 & 1.265 & & 0.904 & 1.069 \\
15 & 0.841 & 1.263 & & 0.862 & 1.066 \\
20 & 0.800 & 1.254 & & 0.825 & 1.062 \\
25 & 0.763 & 1.242 & & 0.791 & 1.058 \\
30 & 0.731 & 1.229 & & 0.760 & 1.053 \\
\hline
\end{tabular}

+ Simple shear: $\square+$ Simple shear: 
TABLE 5. Estimates of the effective shear modulus: three-term Galerkin approximations, $N=4$.

\begin{tabular}{|c|c|c|c|c|c|c|c|c|c|c|}
\hline \multirow{2}{*}{$\begin{array}{l}\text { Void volume } \\
\text { fraction, } f \\
(\%)\end{array}$} & \multicolumn{4}{|c|}{ Simple shear: } & \multirow[b]{2}{*}{$C^{2}$} & \multicolumn{3}{|c|}{ Simple shear: } & $-\square$ & - \\
\hline & $\mu^{*} / \mu$ & $\bar{\varepsilon}_{12}^{*} / \varepsilon_{12}^{0}$ & $C^{0}$ & $C^{1}$ & & $\mu^{*} / \mu$ & $\bar{\varepsilon}^{*} / \varepsilon^{0}$ & $C^{0}$ & $C^{1}$ & $C^{2}$ \\
\hline 5 & 0.940 & 1.268 & 0.994 & 1.324 & -1.198 & 0.950 & 1.064 & 1.074 & 0.088 & -0.125 \\
\hline 10 & 0.887 & 1.269 & 1.025 & 1.184 & -1.073 & 0.903 & 1.069 & 1.092 & 0.073 & -0.130 \\
\hline 15 & 0.840 & 1.266 & 1.070 & 0.992 & -0.913 & 0.862 & 1.067 & 1.097 & 0.068 & -0.135 \\
\hline 20 & 0.799 & 1.257 & 1.066 & 0.981 & -0.909 & 0.825 & 1.063 & 1.096 & 0.062 & -0.134 \\
\hline 25 & 0.763 & 1.244 & 1.074 & 0.918 & -0.864 & 0.791 & 1.058 & 1.092 & 0.057 & -0.128 \\
\hline 30 & 0.730 & 1.232 & 1.085 & 0.840 & -0.806 & 0.760 & 1.054 & 1.083 & 0.064 & -0.128 \\
\hline
\end{tabular}

In Figs. 2 and 3 we have presented the effective bulk and shear moduli, respectively, and compared our results with those of previous investigations, as indicated. ${ }^{7}$ MacKenzie's solution does not include void interaction, whereas the solutions given by Afzali and Nemat-Nasser, while approximate, do include to a certain extent the interaction effects. The present results, on the other hand, take into account the interaction effects completely. It is seen that the void interaction has a strengthening effect on the overall behavior of the solid, as would be expected. The curves in Figs. 2 and 3 labeled NONINTERACTING VOIDS were obtained on the basis of the Eshelby solution for a single void in an infinite medium. In all the above-mentioned results, the Poisson ratio for the matrix is set equal to 0.3 .

It should be noted that the authors mentioned in Figs. 2 and 3 have considered elastic media containing randomly distributed voids or inclusions, whereas here we have examined the case of periodically distributed spherical voids, as also considered by Afzali and Nemat-Nasser [11].

${ }^{7}$ A brief discussion of these solutions is given in Appendix B.

TABLE 6. Estimates of the effective elastic moduli: complete solution, $N=4$.

\begin{tabular}{cccc}
\hline $\begin{array}{c}\text { Void volume } \\
\text { fraction, } f \\
\left(\begin{array}{c}\% \\
\%\end{array}\right)\end{array}$ & $K^{*} / K$ & & \\
& & & \\
\hline 5 & 0.902 & 0.940 & 0.924 \\
10 & 0.828 & 0.887 & 0.868 \\
15 & 0.772 & 0.840 & 0.824 \\
20 & 0.727 & 0.799 & 0.786 \\
25 & 0.692 & 0.762 & 0.753 \\
30 & 0.664 & 0.730 & 0.725 \\
\hline
\end{tabular}




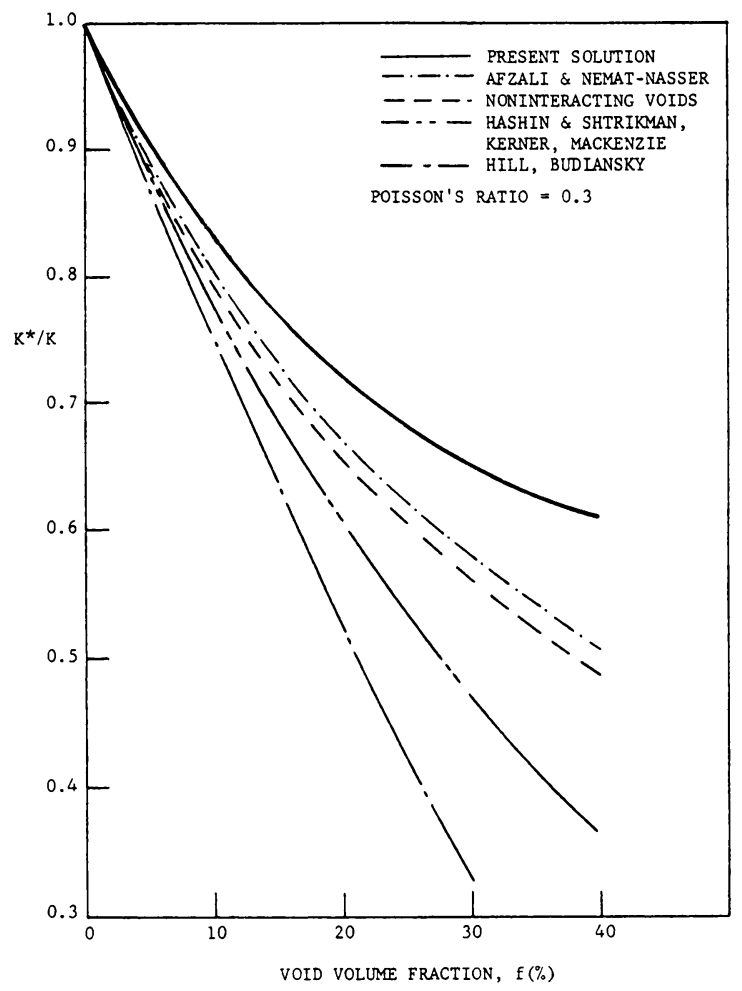

FIG. 2.

Appendix A. To obtain explicit expressions for the functions

$$
g_{\alpha}(\xi)=\int_{\Omega} e^{-i \xi \cdot \mathbf{x}}|\mathbf{x}|^{\alpha} d \mathbf{x}, \quad \alpha=0,1,2
$$

choose the spherical coordinates $r, \theta$, and $\varphi$ in $\Omega$, and observe that

$$
g_{\alpha}(\xi)=\frac{4 \pi}{\xi} \int_{0}^{k} r^{1+x} \sin r \xi d r
$$

Now, from (3.1), and using integration by parts, we arrive for $\xi \neq 0$ at

$$
\begin{aligned}
& g_{0}(\xi)=\frac{4 \pi}{\xi^{3}}(\sin R \xi-R \xi \cos R \xi) \\
& g_{1}(\xi)=\frac{4 \pi}{R \xi^{4}}\left(-2+2 \cos R \xi+2 R \xi \sin R \xi-R^{2} \xi^{2} \cos R \xi\right), \\
& g_{2}(\xi)=\frac{4 \pi}{R^{2} \xi^{5}}\left(-6 \sin R \xi+6 R \xi \cos R \xi+3 R^{2} \xi^{2} \sin R \xi-R^{3} \xi^{3} \cos R \xi\right) .
\end{aligned}
$$




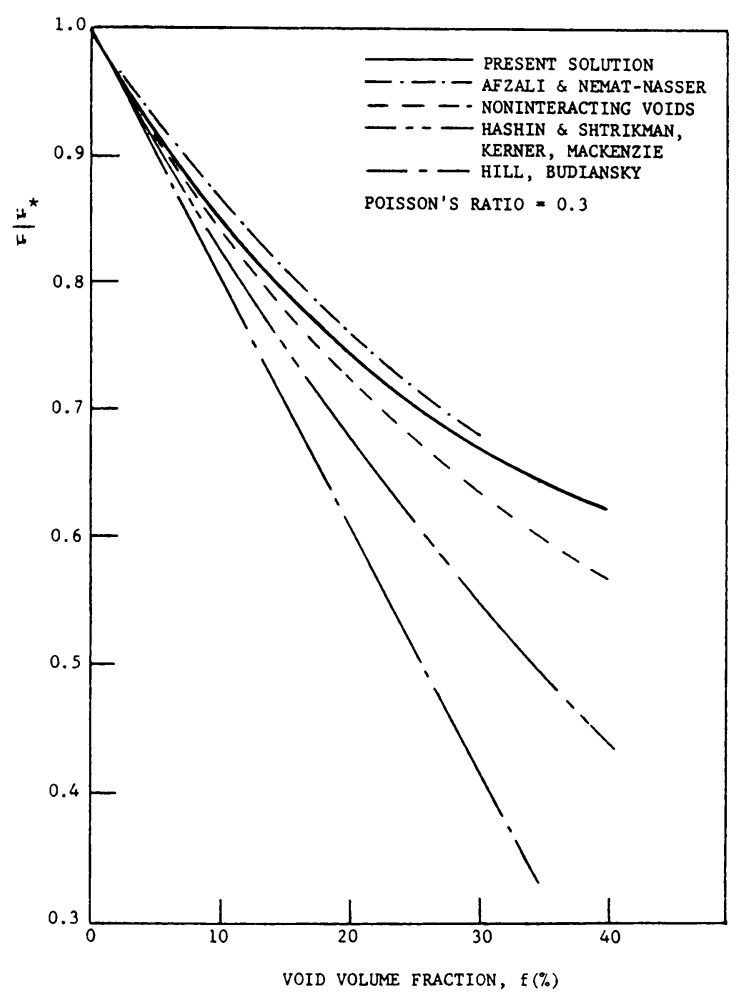

FIG. 3.

Appendix B. The Hashin-Shtrikman bounds are given by Eqs. (4.1)-(4.4) of [3]. For voids and when the Poisson ratio $v$ is taken equal to 0.3 , these bounds become

$$
K_{L}^{*}=\mu_{L}^{*}=0, \quad \frac{K_{U}^{*}}{K}=\frac{1-f}{1+1.625 f}, \quad \frac{\mu_{U}^{*}}{\mu}=\frac{1-f}{1+0.9091 f},
$$

where the subscripts $L$ and $U$ refer to these authors' highest lower bound and the lowest upper bound, respectively. The solution given by Kerner [2], on the other hand, yields

$$
\frac{K^{*}}{K}=\frac{1-f}{1+\frac{3 K}{4 \mu} f}, \quad \frac{\mu^{*}}{\mu}=\frac{1-f}{1+\left(\frac{8-10 v}{7-5 v}\right) f},
$$

which for $v=0.3$ reduce to (B.1) $)_{3,4}$. In a similar way, MacKenzie's solution [1] can be shown to be identical with (B.2) and (B.1) $)_{3,4}$.

The Hill-Budiansky solution [4,5], in the present case, is given by

$$
\begin{gathered}
\frac{f}{1-\frac{3 K^{*}}{3 K^{*}+4 \mu^{*}}}+\frac{1-f}{1+\frac{3 K^{*}}{3 K^{*}+4 \mu^{*}}\left(\frac{K}{K^{*}}-1\right)}=1, \\
\frac{f}{1-\frac{6}{5} \frac{K^{*}+2 \mu^{*}}{3 K^{*}+4 \mu^{*}}}+\frac{1-f}{1+\frac{6}{5} \frac{K^{*}+2 \mu^{*}}{3 K^{*}+4 \mu^{*}}\left(\frac{\mu}{\mu^{*}}-1\right)}=1,
\end{gathered}
$$


which are, in general, coupled equations, requiring a numerical solution. When $v=1 / 2$ (incompressible material), on the other hand, the equations are uncoupled, and one has

$$
\frac{K^{*}}{\mu}=\frac{4(1-2 f)(1-f)}{f(3-f)}, \quad \frac{\mu^{*}}{\mu}=\frac{3(1-2 f)}{3-f} .
$$

In this case, i.e. for $v=0.5$, we have $K^{*}=\mu^{*}=0$ for $f=0.5$.

\section{REFERENCES}

[1] J. K. MacKenzie, The elastic constants of a solid containing spherical holes, Proc. Phys. Soc. 63, $376(1950)$

[2] E. H. Kerner, The elastic and thermo-elastic properties of composite media, Proc. Phys. Soc. 69, 808 (1956)

[3] Z. Hashin and S. Shtrikman, A variational approach to the theory of the elastic behavior of multiphase materials, J. Mech. Phys. Solids 11, 127 (1963)

[4] R. Hill, A self-consistent mechanics of composite materials, J. Mech. Phys. Solids 13, 213 (1965)

[5] B. Budiansky, On the elastic moduli of some heterogeneous materials, J. Mech. Phys. Solids 13, 223 (1965)

[6] J. D. Eshelby, The determination of the elastic field of an ellipsoidal inclusion, and related problems, Proc. Roy. Soc. London A241, 376 (1957)

[7] A. V. Hershey, The elasticity of an isotropic aggregate of anisotropic cubic crystals, J. Appl. Mech. 21, 236 (1954)

[8] E. Kröner, Berechnung der elastischen Konstanten des Vielkristalls aus den Konstanten des Einkristalls, $\mathbf{Z}$. Phys. 151, 504 (1958)

[9] J. R. Willis, Bounds and self-consistent estimates for the overall properties of anisotropic composites, J. Mech. Phys. Solids 25, 185 (1977)

[10] J. Christoffersen, Elastic and elastic-plastic composites: a new approach, DCAMM Report No. 61, The Technical University of Denmark, November 1973

[11] M. Afzali and S. Nemat-Nasser, Radiation effects on elasticity of metals, J. Nuclear Materials, 87, 175 (1979) 